\title{
Convergent Compilation Applied to Loop Unrolling ${ }^{\star}$
}

\author{
Nicholas Nethercote, Doug Burger, and Kathryn S. McKinley \\ The University of Texas at Austin
}

\begin{abstract}
Well-engineered compilers use a carefully selected set of optimizations, heuristic optimization policies, and a phase ordering. Designing a single optimization heuristic that works well with other optimization phases is a challenging task. Although compiler designers evaluate heuristics and phase orderings before deployment, compilers typically do not statically evaluate nor refine the quality of their optimization decisions during a specific compilation.

This paper identifies a class of optimizations for which the compiler can statically evaluate the effectiveness of its heuristics and phase interactions. When necessary, it then modifies and reapplies its optimization policies. We call this approach convergent compilation, since it iterates to converge on high quality code. This model incurs additional compilation time to avoid some of the difficulties of predicting phase interactions and perfecting heuristics

This work was motivated by the TRIPS architecture which has resource constraints that have conflicting phase order requirements. For example, each atomic execution unit (a TRIPS block) has a maximum number of instructions (128) and a fixed minimum execution time cost. Loop unrolling and other optimizations thus seek to maximize the number of mostly full blocks. Because unrolling enables many downstream optimizations, it needs to occur well before code generation, but this position makes it impossible to accurately predict the final number of instructions. After the compiler generates code, it knows the exact instruction count and consequently if it unrolled too much or too little or just right. If necessary, convergent unrolling then goes back and adjusts the unroll amount accordingly and reapplies subsequent optimization phases. We implement convergent unrolling which automatically matches the best hand unrolled version for a set of microbenchmarks on the TRIPS architectural simulator.

Convergent compilation can help solve other phase ordering and heuristic tuning compilation challenges. It is particularly well suited for resource constraints that the compiler can statically evaluate such as register usage, instruction level parallelism, and code size. More importantly, these resource constraints are key performance indicators in embedded, VLIW, and partitioned hardware and indicate that convergent compilation should be broadly applicable.
\end{abstract}

\footnotetext{
* This work is supported by DARPA F33615-03-C-4106, DARPA NBCH30390004, NSF ITR CCR-0085792, NSF CCR-0311829, NSF EIA-0303609, and IBM. Any opinions, findings and conclusions are the authors' and do not necessarily reflect those of the sponsors.
} 


\section{Introduction}

This paper introduces convergent compilation in which the compiler iteratively adjusts its optimization policies based on static self-evaluation.

\subsection{Compiler Phase Ordering and Heuristic Design}

Most compilers include numerous optimization phases. Because finding the optimal code transformation is often NP-complete, most optimizations use heuristic policies. Compiler writers typically tune individual heuristic policies experimentally, based on benchmark behaviors and optimization interactions with previous and subsequent phases. Since phases interact in complex ways, heuristics are not necessarily robust to changes made to another phase or due to phase reordering (e.g., when users specify optimization flags other than the default). To improve the design of individual heuristics, some researchers have turned to machine learning to tune transformation policies [1-3]. To solve the phase ordering problem, compilers have typically relied on a separation of concerns; they postpone handling resource constraints until the end of compilation. For instance, they assume infinite registers for most of compilation and perform register allocation near the end. Most compilers never evaluate during a specific compilation the quality of their upstream predictions. As more resources become constrained, this separation of concerns degrades the compiler's ability to produce high quality code due to the increasing difficulty of predicting how early decisions influence resource constraints.

Increasing hardware complexity is making this problem harder. For instance, shrinking technology increases clock-speed but exposes wire delays, causing less and less of the chip to be reachable in a single cycle [4]. To address this problem, architects are increasingly partitioning resources such as register banks, caches, ALUs (e.g., partitioned VLIW [5-7] and EDGE architectures $[8,9]$ ), and the entire chip (e.g., chip multiprocessors). Partitioning exposes on-chip latencies and resource constraints to the compiler and thus exacerbates the phase ordering problem and makes the separation of concerns solution with no subsequent static evaluation less appealing.

Convergent compilation was motivated by creating blocks full of useful instructions for the block atomic execution model in EDGE architectures. The TRIPS prototype EDGE architecture has a maximum block size of 128 instructions which the architecture maps at runtime on to a grid of 16 arithmetic units which each hold 8 instructions per block $[8,9]$. This array mapping has a fixed overhead. To amortize this overhead and maximize performance, the compiler tries to fill each block with useful instructions while minimizing the total number of blocks. For example, loop unrolling is one method the compiler uses to fill blocks. The compiler performs unrolling early to enable downstream optimizations such as redundant code elimination. However, if it unrolls too much, the resulting code has unnecessary blocks, and if it unrolls too little, each block is less efficient than it could be. Predicting the ideal unroll factor in a block early in the compilation cycle requires modeling down stream optimizations for each loop, and is thus virtually impossible. 
A more conventional unrolling challenge tries to enables redundant code elimination and better scheduling while limiting instruction cache locality degredations and register spilling [10]. Spilling is almost always undesirable, since it increases (1) the number of instructions (loads and stores), (2) latency (two to three cycles in modern caches compared to single cycle register access), and (3) energy consumption due to the cache access. To gain the benefits of unrolling, compilers typically perform it well before register allocation and thus it is difficult for the unroller to predict how its decisions will affect register spilling.

\section{$1.2 \quad$ Improving Optimization Quality}

Previous solutions to solving the phase interaction problem include static evaluation of inlining's effect on other optimization phases [11] and iterated register allocation and instruction scheduling to resolve their tensions [12]. Both of these approaches require all participating phases to annotate and encode the results of their decisions. Thus, each phase must be appropriately engineered for all interacting optimizations, and must be cognizant of their phase ordering and influence on other optimization passes. Convergent compilation simplifies and generalizes over these approaches.

Instead of trying to model and perfect all the heuristics and phase interactions, convergent compilation improves one or more optimization heuristic by statically evaluating decisions after performing other interacting phases. If necessary, it then adjusts the heuristic for the particular code fragment and reoptimizes. More formally, given a sequence of ordered compilation phases $\left\{P_{1}\right.$, $\left.P_{2}, \ldots, P_{n}\right\}, P_{i}$ records its optimization decisions. After some later phase $P_{k}$, $k>i$, a static evaluation phase $P_{E v a l}$ measures the effectiveness of $P_{i}$. If $P_{E v a l}$ decides $P_{i}$ 's decisions were poor, the compiler uses a checkpoint/rollback mechanism to reapply a modified $P_{i}$ and subsequent phases by feeding back information from $P_{\text {Eval }}$ 's static evaluation to help $P_{i}$ adjust its heuristic and do a better job. To minimize the additional compile time, the compiler may choose a single optimization and iterate once, or to apply iteration only to key methods. To more thoroughly explore code quality sensitivities, the compiler may iterate on several key optimizations. Given repeated compilation of the same code, a repository could eliminate some of this overhead [13], or could enable the compiler to explore many phase interactions incrementally with modest per-compile overheads.

For example, in the TRIPS compiler we implement convergent unrolling $\left(P_{i}\right)$ which checkpoints an intermediate representation, and records how many times it unrolls each loop. After other optimizations, the static evaluation phase $\left(P_{\text {Eval }_{i}}\right)$ counts the instructions in a block. Assume the best unroll amount is $n$, if the compiler unrolls too little, $P_{E_{\text {Eval }}}$ will go back and unroll more, if it unrolls too much, $P_{\text {Eval }_{i}}$ will go back and unroll less. To roll back, the compiler discards the code fragments in question, reloads the checkpoint, improves the unroller's heuristic with the $P_{E v a l}$ feedback, invokes unrolling and subsequent phases.

Transformations that are suited to this approach must simply and statically record their decisions, and their corresponding evaluation function, $P_{\text {Eval }}$, must statically evaluate the resulting code quality. In unrolling, $P_{i}$ records the number 
of iterations it unrolls, and the code generator (or a separate $P_{\text {Eval }}$ phase) counts the instructions in a block. Suitable convergent optimizations include any that increase or change usage of limited resources (such as inlining, unrolling, register allocation, and scheduling), and have simple evaluation functions (such as instruction count, registers, loads/stores, or code size).

This paper makes the following contributions.

- Section 3 presents a convergent compilation model that simplifies and generalizes previous self-evaluating approaches. It mitigates the problem of selecting the perfect heuristic and phase ordering. It is suitable for both ahead-oftime and just-in-time compilation.

- Sections 4 and 5 shows convergent unrolling is effective for the TRIPS processor which is particularly sensitive to choosing the right unroll factor.

- Section 6 lists additional compiler transformations suited to this approach, and a useful variation called convergent cloning.

Although convergent compilation is motivated by our TRIPS compilation problems, it is applicable in many more settings. For instance, embedded programs have strict instruction space requirements and limited register files. Compilers for embedded processors could more easily assess the costs and benefits of code expanding (e.g., inlining, unrolling) and code size reduction (e.g., procedure abstraction, inlining) transformations with this approach.

\section{Related Work}

This section compares convergent compilation with related work on selecting good optimization heuristics, phase ordering, and other compiler feedback loops.

\subsection{Designing Optimization Heuristics}

The difficulty of designing good heuristics for individual optimizations is witnessed by a diversity of advanced approaches that to automate this process and search the optimization space $[1-3,14,15]$. For example, Cavazos and Moss use supervised learning to decide whether to schedule blocks in a Java JIT [3] and Cavazos et al. use code features to select between a linear scan or graph coloring register allocator [16]. Stephenson et al. use genetic algorithms to derive hyperblock formation, register allocation, and prefetching; and supervised learning for unrolling $[1,2]$.

One difficulty for the learning algorithms is that they currently hold the rest of the compiler constant, thus learning one heuristic at a time. The convergent approach more naturally responds to changes in the other phases (which are numerous in our research compiler). This advantage may decrease in a production setting where phases change less frequently. Although we do not evaluate it, it may be possible to learn an unrolling heuristic that produces similar code quality to convergent unrolling. However, it is more likely that these approaches are complementary. Convergent compilation may produce better code starting with a "one-size-fits-all" learned heuristic and then further specializing for the specific program. Prior results that compare self and cross program learning [3, $1,2]$ indicate that this specialization will improve performance. 


\subsection{Phase Interactions}

The most closely related work performs static self-evaluation, but is not as general convergent compilation or requires more pervasive changes to the compiler $[11,12]$. Brasier et al. use a static feedback loop to reduce the antagonism between instruction scheduling and register allocation in a system called CRAIG [12]. It first performs instruction scheduling followed by register allocation; if it spills too much, it starts over and does register allocation before performing incremental instruction scheduling, moving towards late assignment. CRAIG thus reorders phases and refines its scheduling heuristic based on spill feedback. Convergent compilation generalizes beyond these two closely related phases by communicating more information across more phases.

Dean and Chambers use inlining trials to avoid designing a heuristic that models the exposed optimization opportunities for subsequent phases [11]. After inlining, each subsequent phase must carefully track how inlining influenced its decisions. If inlining does not enable optimizations that reduce the resulting code size (a static measure), the compiler reverses the inlining decision, recompiles the caller, and records the decision to prevent future inlining of this method and similar ones. This approach is limited because it requires changes to all subsequent phases for each optimization it wishes to evaluate. Section 6 describes how convergent inlining eliminates the need to change all intervening phases.

\subsection{Iterative Compilation}

Iterative compilation seeks to evaluate the extent of the phase ordering problem and to improve over default orderings. Iterative compilation reorders, repeats, and omits phases to empirically evaluate hundreds or thousands of compileexecute sequences to find the configuration that produces the fastest or smallest code for a particular program. Results show that default compiler phase orderings and settings are far from optimal [17-22]. Because the configuration space is huge, researchers use search techniques such as genetic algorithms and simulated annealing to reduce the number of compile-execute cycles and still attain benefits. For example, Lau et al. use performance measurement to select the best performing among a small set of pre-computed optimization strategies [23].

Convergent compilation is more robust to compiler changes (e.g., changing a policy or adding a heuristic), whereas iterative compilation must perform all the trials after any changes. However, convergent compilation could be a component of a system that attains benefits of phase selection and heuristic tuning at a reduced cost by using static objective functions. In fact, Triantafyllis et al. propose a compiler optimization-space exploration system that uses an iterative approach, but aggressively prunes the program to its most critical components based on dynamic behavior and then compares program versions using static performance estimation to reduce compile-time [22]. Performance estimation is a general approach. Convergent compilation instead couples optimization objective functions which are easy to measure with the final code quality on key resources such as register usage, instruction counts for blocks, and instruction level parallelism. Although we demonstrate only instruction counts for blocks 
with unrolling, many heuristic objective functions, such as adding spills to the register allocator is correlated with performance.

\subsection{Feedback-Directed Optimization}

Smith defines feedback-directed optimizations (FDO) $[24-27,13]$ as a family of techniques that alter a program's execution based on tendencies observed in its present or past runs. FDO is orthogonal to convergent compilation since FDO typically uses dynamic edge, path, or method profiling to select which code to optimize, and to guide heuristics. Arnold et al. add a repository to combine online and offline (previously profiled) optimization plans that incorporate the costs and benefits of online optimization [13]. Convergent compilation instead feeds back static evaluations, e.g., code size or register usage, and then adjusts heuristics to avoid phase ordering problems, all without ever executing the program. Convergent compilation and FDO are thus complementary.

\section{Convergent Compilation}

This section describes convergent compilation in two parts. First, it explains its most general form for use in an ahead-of-time compiler. Second, it discusses three specific instances of the general approach, with different simplicity and compile-time trade-offs.

\subsection{Convergent Compilation in the Abstract}

Convergent compilation involves the following compiler phases.

$P_{C P}$ : Checkpoints code fragments (e.g. saves them to memory or file).

$P_{i}: \quad$ Transforms/optimizes the code using a heuristic.

$P_{E v a l}$ : Evaluates the effectiveness of the $P_{i}$ transformation.

$P_{L B}: \quad$ Determines if the current code fragment is acceptable or should be recompiled with a modified heuristic, based on the results of $P_{\text {Eval }}$ and the number of times $P_{i}$ has executed.

$P_{R B}:$ Rolls back to a checkpointed code fragment.

Figure 1 gives the pseudocode for the most general form of convergent compilation. Figure 2 shows its control flow and data flow in a diagram. The approach involves two main loops. The inner loop is the heart of convergent compilation. It processes one code fragment at a time, where a fragment could be a loop, a procedure, or a module. It performs the transformation phase $P_{i}$ and subsequent phases, then runs $P_{E v a l}$ to evaluate $P_{i}$ 's decisions. It uses $P_{R B}$ to roll back the fragment to the version checkpointed by $P_{C P}$ if necessary, and repeats until the fragment $F$ is deemed "good enough," or it reaches a limit on its number of iterations (shown as the edge that skips $P_{E v a l}$ ). The outer loop iterates through the code fragments one at a time.

$P_{i}$ and $P_{\text {Eval }}$ interact in three key ways. First, $P_{i}$ must indicate to $P_{\text {Eval }}$ what decisions it made. $P_{i}$ annotates the code with this information. In our example, $P_{i}$ identifies unrolled loops and their unroll factor. Second, $P_{E v a l}$ must be able to meaningfully evaluate $P_{i}$ 's decisions. $P_{\text {Eval }}$ must include a static measurement that evaluates code quality. A simple example is "this unrolled loop 


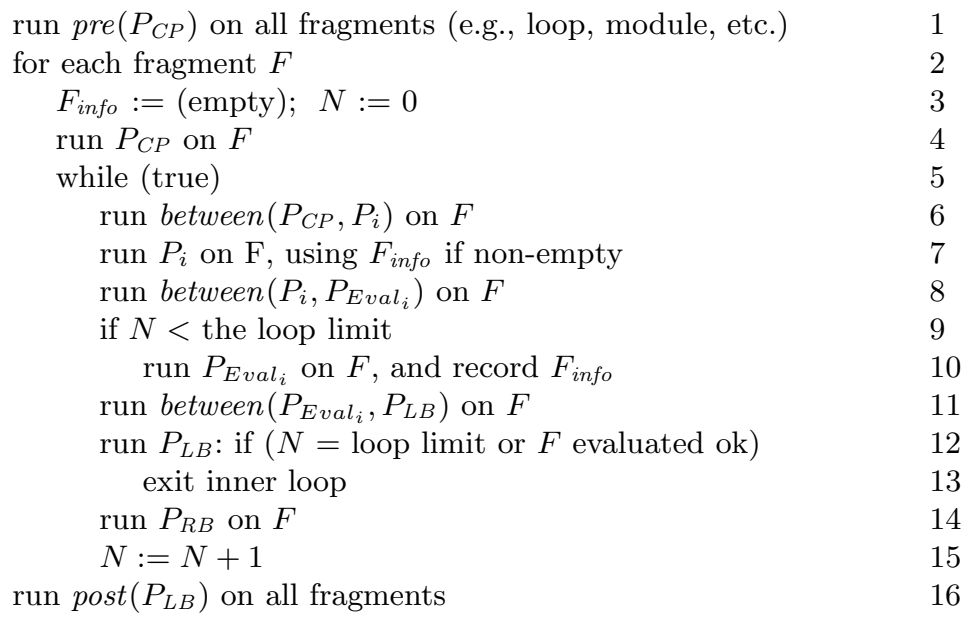

Fig. 1. Convergent Compilation Pseudocode. pre $(P)$ gives the phases that precede $P$; between $(P, Q)$ gives the phases between $P$ and $Q ; \operatorname{post}(Q)$ gives the phases that come after $Q$.

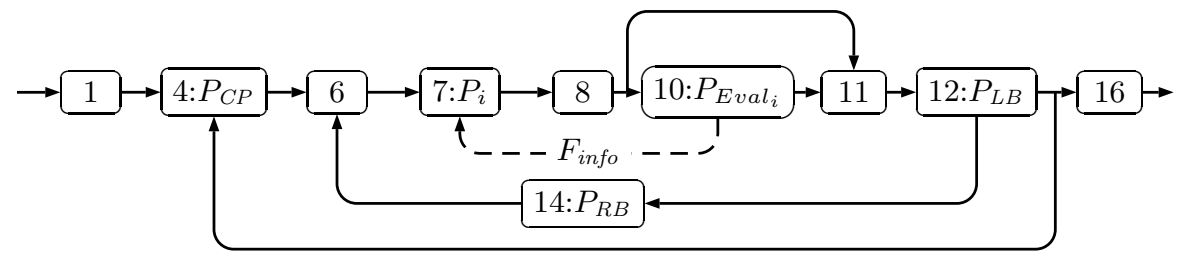

Fig. 2. Control flow of convergent compilation. Solid lines represent control flow, dashed lines represent data flow. The number labels correspond to pseudocode line numbers in Figure 1.

has too many spills." Any performance improvements depend on the accuracy of $P_{E v a l}$ 's evaluations. Third, $P_{E v a l}$ should help $P_{i}$ improve its decisions on any rejected fragment. In our example, $P_{E v a l}$ could indicate that the chosen unroll factor was too high (e.g., more blocks than necessary and an under-full block) or too low (e.g., one under-full with room for more unrolled iterations), or just right. If needed, the unrolling heuristic adjusts the unroll factor accordingly. Figure 2 depicts this information flow with a dashed line.

Thus far we have discussed using one $P_{i} / P_{\text {Eval }}$ pair, but the compiler can use more. For example, one could employ a single large inner loop: perform all the $P_{i}$ transformations on a fragment, then evaluate each $P_{E v a l}$, and if any transformations were unsatisfactory, loop back and adjust them. This structure requires only checkpointing once, but performs all $P_{i}$ phases even if only a single $P_{\text {Eval }}$ evaluation failed. Alternatively, one could employ more checkpointing and have multiple inner loops, which might overlap or be entirely separate. The best configuration would depend on how the different $P_{i}$ phases interact and the increase in compile time the system is willing to tolerate. 


\subsection{Instantiating Convergent Compilation}

We can partially or fully instantiate this general convergent compilation framework by specifying some or all of its parameters: the size of each fragment $F$, the loop limit $N$, the workings of the phases $P_{i}, P_{E v a l_{i}}, P_{C P}, P_{R B}, P_{L B}$, and the phases present in each of pre $\left(P_{C P}\right)$, between $\left(P_{C P}, P_{i}\right)$, between $\left(P_{i}, P_{\text {Eval }_{i}}\right)$, between $\left(P_{E_{\text {val }}}, P_{L B}\right)$ and post $\left(P_{L B}\right)$. The following paragraphs describe three such general instances of a single convergent optimization, and Section 4 presents convergent unrolling.

A complex but efficient instance of a convergent optimization. Code fragments are procedures, and the inner loop executes at most twice. $P_{C P}$ and $P_{R B}$ are simple: $P_{C P}$ saves a copy of a procedure in memory, and $P_{R B}$ discards the poorly optimized code and reverts to the saved code. The phase sequences between $\left(P_{C P}, P_{i}\right)$ and between $\left(P_{E_{\text {val }}}, P_{L B}\right)$ are empty. We do not specify the other parameters- $P_{i}, P_{\text {Eval }_{i}}, \operatorname{pre}\left(P_{C P}\right)$, $\operatorname{between}\left(P_{i}, P_{E v a l}\right)$ and $\operatorname{post}\left(P_{L B}\right)$ - so this instance still has some generality.

This instance is efficient - it minimizes the number of phases that execute repeatedly, and the small fragment size reduces the cost of individual phase times and the size of the checkpoint. However, it requires some structural support in the compiler, e.g., the ability to run multiple phases in succession on a single procedure. Procedures are a good general choice for fragments as they are typically not too big, but are still stand-alone units. Smaller units are possible, but require more work to merge fragments. The efficiency of this instance depends on how often $P_{i}$ makes bad decisions; if it makes no bad decisions, it will not iterate at all.

A simpler instance. A variant of the first instance involves changing the size of the code fragments to an entire module, which effectively removes the outer loop. This structure is less efficient - checkpointing and possibly repeating phases for the entire module - but simpler to implement. Note that although the fragment size is a whole module, the heuristics are adjusted at a finer level. For example, with loop unrolling $P_{E v a l}$ will include information about every unrolled loop in $F_{\text {info }}$. If any of the loops in the module are rejected by $P_{L B}$ the whole module is recompiled. As a result, the fragment size does not affect accuracy, only the amount of checkpointing and number repeated phases.

An easy-to-implement instance. Figure 3 shows a simple way to implement this approach in which the compiler executes twice. In the first pass $P_{E v a l}$ writes $F_{\text {info }}$ for the whole module to a file. In the second pass $P_{i}$ reads $F_{\text {info }}$ from this file and adjusts its decisions accordingly and $P_{\text {Eval }}$ does not execute. Each code fragment is an entire module. $P_{C P}$ and $P_{R B}$ are no-ops; since the compiler runs twice, the original source code serves as the checkpoint. Both pre $\left(P_{C P}\right)$ and $\operatorname{post}\left(P_{L B}\right)$ are empty.

This instance is an excellent way to trial convergent compilation in an existing compiler, since the only change needed is support for writing and reading the data file; a wrapper script can implement the inner loop. We implemented convergent unrolling exactly this way for our evaluation in Section 5 . This structure sacrifices efficiency for simplicity by doubling compilation time. 


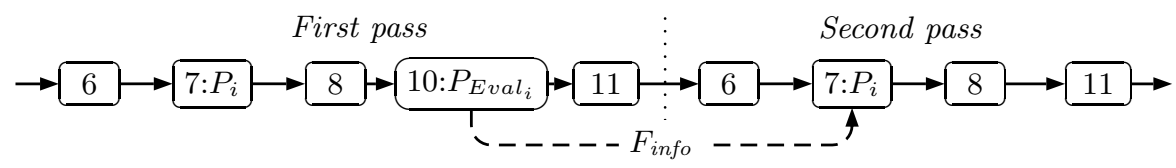

Fig. 3. Control flow of an easy-to-implement instance of convergent compilation. Solid lines represent control flow, dashed lines represent data flow. The number labels correspond to pseudocode line numbers in Figure 1.

\section{Convergent Loop Unrolling for TRIPS}

In this section, we review loop unrolling, describe the TRIPS architecture and the unique challenges it poses to the loop unroller, and explain how convergent unrolling helps to solve these problems.

\subsection{Loop Unrolling}

Loop unrolling is a common transformation which duplicates a loop's body one or more times. The unroll factor is the number of copies of the loop body in the final unrolled loop; an unroll factor of one means no unrolling. The simplest case is when the loop trip count is known statically and the unroll factor divides it evenly; the unrolled loop on the right has an unroll factor of three.

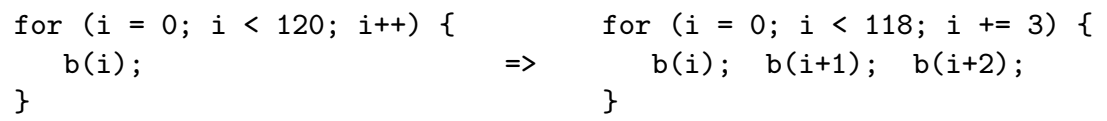

We restrict this discussion to loops with sufficiently large trip counts, but the same framework flattens loops with small trip counts. If the loop trip count is known statically but the unroll factor does not divide it evenly, or the loop trip count is statically unknown but invariant, a "clean-up" loop performs the final few iterations. If the trip count is known statically, the clean-up loop can be flattened.

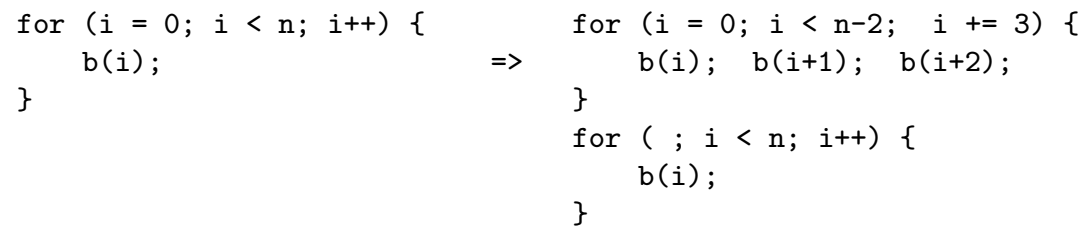

Loop unrolling can improve program performance on traditional architectures in two ways. First, the unrolled loop requires fewer instructions, because there are fewer loop tests and backward branches, and in some forms, fewer updates of the index variable. On modern architectures, branch prediction and multiple issue mitigate these benefits, but the TRIPS block limit on instructions does benefit. Second and more importantly, unrolling enables other optimizations. For example, if the body loads or stores the same memory location on distinct adjacent iterations, scalar replacement can replace some memory accesses with register 


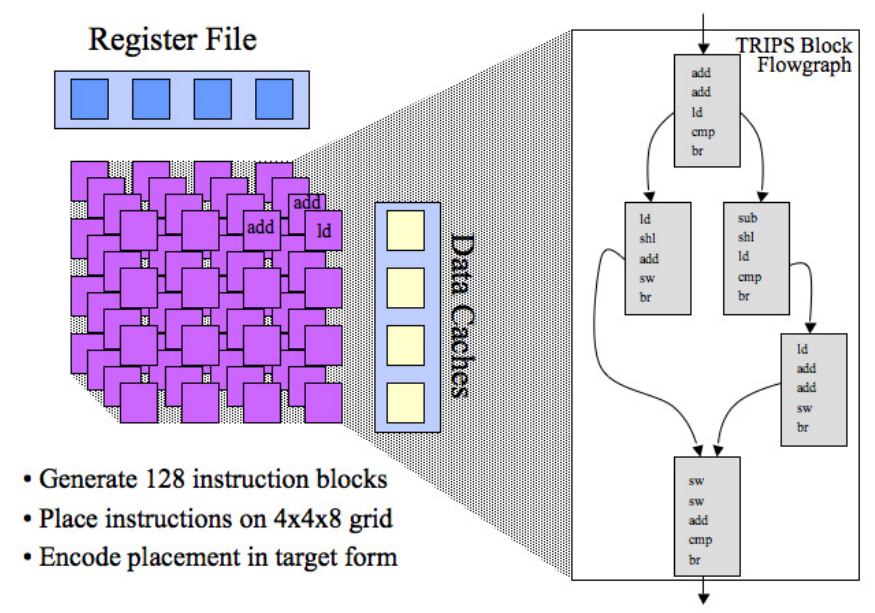

Fig. 4. The TRIPS prototype compilation target.

accesses. Loop unrolling also exposes instruction level parallelism (ILP) to static and dynamic instruction scheduling. The cost/benefit analysis for unrolling must also include the hard-to-model effect on code size which if increased too much degrades instruction cache performance, and increases in register pressure which cause spilling.

\subsection{TRIPS}

TRIPS is a prototype implementation of a new class of microprocessor architectures called EDGE (Explicit Data Graph Execution) designed to provide high performance and low power consumption in the face of technology trends such as increasing clock speed and increasing wire delays $[8,9,28]$. The prototype design is complete and working chips should be operational in 2006 .

Unlike traditional architectures that operate at the granularity of a single instruction, EDGE architectures implement serial, block-atomic execution, mapping a block of instructions on to the ALU grid (as depicted in Figure 4), executing it atomically, committing it, and fetching the next block. Execution within blocks is dataflow; each instruction forwards its results directly to its consumers in that block without going through a shared register file (registers are only used for inter-block value passing). Each block orders its memory references to insure sequential consistency. Each block is a hyperblock - a single-entry, multiple-exit set of predicated basic blocks $[29,30]$ — with some additional constraints [8].

In the TRIPS prototype, a key constraint is that each block is fixed-size and holds at most 128 instructions. Each ALU in the $4 \times 4$ ALU grid has up to 8 instructions in each block mapped onto it $(4 \times 4 \times 8=128)$. Up to 16 instructions can execute per cycle, one per ALU. Up to 8 blocks can be in-flight at once ( 7 of them speculatively), resulting in a 1,024-wide instruction window.

The biggest challenge for the TRIPS compiler [9] is to create blocks full of useful instructions; ideally it will produce high quality blocks with close to 128 instructions, maximizing ILP and minimizing the fixed per-block execution over- 
heads. To achieve this goal, the compiler's main tools are the use of predication to include multiple basic blocks in each TRIPS block (hyperblock formation), inlining, and loop unrolling. The TRIPS compiler also uses convergent hyperblock formation to create full blocks: it incrementally merges blocks, optimizes them, and if too full, rolls back [31].

\subsection{Challenges for Loop Unrolling on TRIPS}

To maximize TRIPS performance, the loop unroller would ideally produce loops containing exactly, or slightly fewer than, 128 instructions. Consider a loop with a known trip count of 120 , in which each loop body has 31 instructions, and the loop test and exit are 4 instructions. If the unroll factor is 3 , the block size is $31 \times 3$ $+4=97$ instructions, and the loop will execute in 40 blocks. However, an unroll factor of 4 yields a block size of $31 \times 4+4=128$ instructions, and the loop will execute in only 30 blocks. If the size of the loop body is 32 instructions instead, an unroll factor of four produces a loop size of 132 instructions which requires two TRIPS blocks, and 60 block executions. An unroll factor of 2 produces this same result.

This example demonstrates that accurate instruction counts are vital for loop unrolling on TRIPS; in some cases, even underestimating the size of a loop body by one instruction can harm code quality. This example assumes that all duplicated loop bodies have the same size, but downstream optimizations like common subexpression elimination and test elision change the resulting code size further complicating the job of estimating the final number of instructions. The TRIPS compiler performs unrolling early in the optimization sequence because many optimizations, such as scalar replacement, further improve code quality after unrolling. Accurate instruction counts are not available to the unroller and become available only near the end of compilation.

\subsection{Convergent Loop Unrolling}

To solve this problem, we use a simple two-pass approach, as described in Section 3.2. In the first pass, the compiler performs the phases pre $\left(P_{i}\right)$ (represented by box 6 in Figure 3), which include parsing, inlining, and conversion to a static-single assignment, control-flow graph-based intermediate representation (IR). The loop unroller $\left(P_{i}\right)$ then executes for the first time. For each candidate loop, it estimates the number of TRIPS instructions in the loop body $\left(S_{B}\right)$, and the loop and exit tests $\left(S_{E}\right)$ by examining each IR instruction in the loop. If $S_{B}+S_{E}>128$, no unrolling takes place, otherwise it selects an unroll factor of $U_{1}=\left(128-S_{E}\right) / S_{B}$. Loops that are larger than half a block are therefore never unrolled; we have experimented with trying to unroll to fit three loop bodies in two blocks, but with little success. One exception is that the unroller flattens loops with a known loop bound up to 512 instructions. Because flattened loops have no back edges, subsequent phases can easily merge them with surrounding blocks.

After unrolling, $P_{i}$ marks the unrolled loops with the estimated sizes. The compiler then performs the phases in between $\left(P_{i}, P_{\text {Eval }}\right.$ ) (box 8 in Figure 3), which in the TRIPS compiler include many optimizations (scalar replacement, 
constant propagation, global value numbering, etc.), code generation, and hyperblock formation. $P_{\text {Eval }}$ then measures the actual sizes of the unrolled loopsaccurate measurements are possible in this phase-and writes its measurements $\left(F_{\text {info }}\right)$ to the data file. The final phases in $\operatorname{post}\left(P_{\text {Eval }_{i}}\right)$ then execute which include block splitting, register allocation, and instruction scheduling [9].

The second pass is like the first except $P_{i}$ does not use IR-based loop size estimation. Instead, for each unrolled loop it reads from the data file $\left(F_{\text {info }}\right)$ the measured size of the entire unrolled loop $\left(S_{L}\right)$ and the loop test and exit $\left(S_{E 2}\right)$. It then estimates the size of each loop body as $S_{B 2}=\left(S_{L}-S_{E 2}\right) / U_{1}$. This estimate is imperfect because all iterations in an unrolled loop are not always exactly the same size, but it improves over the IR-based estimate (Section 5.1 quantifies the difference). With this more accurate loop size estimate, it can now compute the new unroll factor, $U_{2}=\left(128-S_{E 2}\right) / S_{B 2}$, and compilation continues to the end.

Convergent unrolling results in fuller TRIPS blocks, reducing the block execution count and speeding up programs, as the next section shows.

\section{Evaluation}

This section evaluates convergent unrolling's effectiveness in improving loop unrolling for TRIPS. Because TRIPS hardware is not yet available, we use a simulator for our experiments. It is cycle-accurate and slow, so we use only microbenchmarks for our evaluation. The suite consists of 14 microbenchmarks containing key inner loops extracted from SPEC2000, five kernels from an MIT Lincoln Laboratory radar benchmark (doppler_GMTI, fft2_GMTI, fft4_GMTI, transpose_GMTI, forward_GMTI), a vector add kernel (vadd), a ten-by-ten matrix multiply (matrix_1), and a discrete cosine transform (dct8x8). Because these benchmarks are all dominated by loops, the unrolling benefits should be large.

\subsection{Goals}

Convergent unrolling statically measures how full blocks are to dynamically reduce the number of blocks and thus execution time. Convergent unrolling therefore has three goals: 1) to improve the size estimates and thus produce fuller blocks; 2) to execute fewer blocks at runtime because each block is fuller; and thus 3) to speed up the program by a) exposing and exploiting more ILP within a block and b) by fetching fewer blocks and thus reducing the time required to map blocks onto the ALU grid.

We measure static block size estimates, dynamic block executions, and execution times. We use as our straw-man unrolling by 3 which produces 4 loop bodies because we and others (e.g., gcc and the Alpha GEM compiler) have found it is a simple and reasonable heuristic for conventional architectures. We use the best intermediate representation (IR) estimator we were able to construct for our base TRIPS unroller, and then apply convergent unrolling to refine this estimator.

\subsection{Results}

Static Block Size Estimates. Convergent unrolling works well in improving the unroller's estimates of loop sizes, the first goal. The microbenchmark suite has 


\begin{tabular}{|c|c|c|c|c|c|c|c|c|}
\hline & \multicolumn{8}{|c|}{ Unrolling Policies } \\
\hline & \multicolumn{4}{|c|}{ Block Count Reduction } & \multicolumn{4}{|c|}{ Cycle Count Reduction } \\
\hline & & $\%$ & $\%$ & $\%$ & & $\%$ & $\%$ & $\%$ \\
\hline & none & by 3 & Est & $\mathrm{CU}$ & none & by 3 & Est & $\mathrm{CU}$ \\
\hline $\begin{array}{l}\text { art_2 } \\
\end{array}$ & 22061 & 66.3 & 66.3 & 84.9 & 504565 & 61.6 & $\begin{array}{l}61.6 \\
\end{array}$ & 79.1 \\
\hline vadd & 54334 & 84.0 & 84.1 & 84.1 & 439449 & 71.9 & 73.3 & 73.3 \\
\hline transpose_GMTI & 78349 & 83.8 & 83.8 & 83.8 & 1027366 & 75.4 & 75.5 & 75.5 \\
\hline matrix_1 & 24665 & 81.5 & 81.5 & 81.5 & 383814 & 68.7 & 68.7 & 68.7 \\
\hline art_3 & 30051 & 49.9 & 76.8 & 80.9 & 443259 & 47.8 & 70.0 & 68.9 \\
\hline art_1 & 16651 & 64.3 & 66.2 & 78.9 & 233755 & 50.5 & 56.7 & 67.4 \\
\hline twolf_1 & 38631 & 53.7 & 65.3 & 76.6 & 689344 & 57.7 & 58.4 & 62.7 \\
\hline twolf_3 & 14051 & 49.8 & 49.7 & 66.3 & 673539 & 2.3 & 2.8 & 6.3 \\
\hline gzip_1 & 2395 & 32.9 & 54.7 & 57.3 & 24664 & -10.1 & -5.8 & -2.3 \\
\hline bzip2_2 & 32911 & 44.7 & 51.7 & 52.5 & 426155 & 31.4 & 38.8 & 41.0 \\
\hline bzip2_1 & 15682 & 0.2 & 0.2 & 49.6 & 410409 & 30.9 & 31.2 & 39.5 \\
\hline doppler_GMTI & 11190 & 43.8 & 12.5 & 37.5 & 396943 & 21.0 & 15.7 & 24.4 \\
\hline equake_1 & 16405 & 62.2 & 37.3 & 37.3 & 331378 & 55.1 & 43.9 & 43.9 \\
\hline gzip_2 & 8986 & 40.6 & 30.2 & 30.2 & 129110 & 48.5 & 31.7 & 31.7 \\
\hline forward_GMTI & 11825 & -5.1 & 13.5 & 13.5 & 392571 & 11.0 & 14.7 & 14.7 \\
\hline ammp_2 & 30951 & 5.5 & 16.5 & 11.0 & 910693 & 32.1 & 36.0 & 34.7 \\
\hline ammp_1 & 60751 & 0.0 & 8.9 & 8.9 & 1891762 & 0.0 & -8.1 & -8.1 \\
\hline $\operatorname{dct} 8 x 8$ & 3046 & 9.2 & 4.2 & 4.2 & 61756 & 11.8 & 41.2 & 41.2 \\
\hline parser_1 & 7051 & -33.0 & 0.0 & 0.0 & 225255 & 11.6 & 6.1 & 0.0 \\
\hline bzip2_3 & 15531 & 0.0 & -48.3 & 0.0 & 400906 & 14.0 & -1.6 & 0.0 \\
\hline fft4_GMTI & 9745 & -9.2 & -8.2 & -8.2 & 142233 & -8.8 & -10.6 & -10.6 \\
\hline fft2_GMTI & 8378 & -23.3 & -9.5 & -9.5 & 245022 & -10.9 & -2.7 & -2.7 \\
\hline arith. mean & & 31.9 & 33.5 & 41.9 & & 30.6 & 31.7 & 34.1 \\
\hline
\end{tabular}

Table 1. Block and cycle results for unrolling. Column 1 gives the benchmark name. Column 2 (none) gives the number of blocks executed with no unrolling. Columns 3-5 give the percentage reduction in the number of blocks executed vs. no unrolling: column 3 (by 3 ) is default unrolling, column 4 (Est) is IR size estimation only, column 5 (CU) is convergent unrolling. Columns 6-9 give the corresponding cycle results.

33 candidate loops. On the first pass, using the IR-based loop size estimator, the average relative error of this estimate was $61 \%$. The errors include both under and overestimates. On the second pass, using the back end measurements of the resulting loop sizes, the average relative error was $6 \%$. Most of the estimates by the second pass were within 1 or $2 \%$; the worst result was a $24 \%$ underestimate. Poor final estimates correlated with the worst initial estimates, since the unroll factor chosen in the second pass was quite different from the first pass, and thus there was more room for error. Performing this cycle a third time attains small improvements, but is probably not worth the extra compilation time.

Dynamic Block and Cycle Results. Table 1 quantifies how well convergent unrolling achieves the second and third goals. We perform the following four experiments.

- No unrolling (none). 
- Default unrolling (by 3): unroll by three for unknown loop bounds; for known bounds, flatten loops if the flattened size is fewer than 200 statements; otherwise choose an unroll factor based on an IR estimate.

- IR code estimation (Est): the compiler estimates the number of TRIPS instructions from the IR.

- Convergent unrolling corrected estimation (CU): uses the two-pass compiler structure and phases described above.

On average, convergent unrolling decreases the average number of blocks executed by $10 \%$ over the default unroller, and $8 \%$ over IR estimation (Est). It attains this result by substantial improvements over default unrolling on art_2, art_3, twolf_1, twolf_3, and bzip2_1, and avoiding the substantial degradations of IR estimation and default on parser_1, bzip2_3, and fft2_GMTI. For art_2 and art_3 the improvement is due to convergent unrolling using higher unroll factors ( 6 and 8 instead of 3 ) for loops with small bodies; for the others the improvement is caused by choosing lower unroll factors (e.g. 2 instead of 3,3 instead of 6 ) for loops with larger bodies, so that the entire unrolled loop fits within one block. IR estimation is only marginally better than the default non-TRIPS-specific policy, which shows that the TRIPS-specific unrolling policy is of little use without convergent unrolling's accurate size estimates.

Although block counts are a good indicator of performance, they are not perfect (e.g., gzip_1 and ammp_1). The cycle count improvements with convergent unrolling are thus smaller than the block improvements. This result is due to architectural details that are beyond the scope of this paper. However, these results are more encouraging than they seem. Currently the TRIPS compiler does not perform instruction-level hyperblock optimizations, so the code it produces still has some "fat". Experiments with hand-coded microbenchmarks show that cycle count improvements due to loop unrolling increase as code quality goes up. For example, we have seen that better scalar replacement reduces twolf_3's run-time by around $20 \%$.

Taken together, the dynamic block size and block execution results demonstrate that convergent unrolling can help bridge the phase ordering problem and provide improved heuristics for loop unrolling.

\section{Discussion}

This section describes other potential convergent optimizations, mechanisms for mitigating increases in compilation time, and how to integrate convergent compilation into a JIT compiler.

\subsection{Other Convergent Optimizations}

This section describes other optimizations that interact with resource usage and are thus amenable to static evaluation.

- Loop unrolling can also increase register spilling. $P_{\text {Eval }_{i}}$ could decide to reject any loop in which unrolling causes a register spill. 


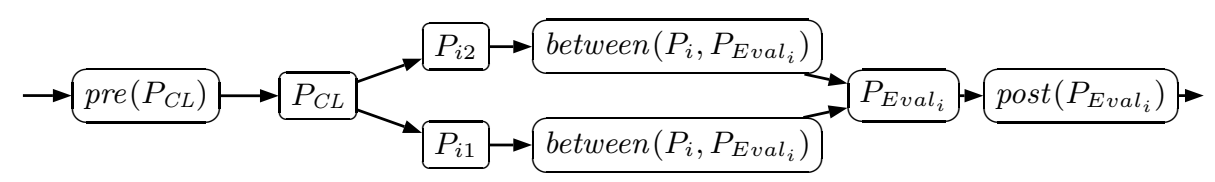

Fig. 5. Control flow of convergent cloning.

- We are also using this approach for convergent hyperblock formation in the TRIPS compiler [31]. When considering the inclusion of multiple basic blocks for a single TRIPS block, it is difficult to estimate the resulting block size beforehand because of subsequent optimizations (just like unrolling for block size). Instead, we optimistically and incrementally combine blocks, run the optimizations on the the resulting hyperblock, and roll back if it exceeds 128 instructions.

A variant we call convergent cloning removes the feedback loop but still uses static evaluation. Its control flow is shown in Figure 5. Instead of $P_{C P}$ checkpointing the code, a cloning phase $P_{C L}$ makes one or more temporary clones of a code fragment. $P_{i}$ then operates in a different way on each clone. After the intervening phases operate on each clone, $P_{E v a l}$ then statically compares the clones and chooses the best one, discarding the others. This approach is most useful when $P_{i}$ can choose between only a small number of possible transformations (e.g. whether to inline a particular procedure call or not). The following list gives some cases where this variation might be applicable.

- If $P_{i}$ is an inliner, $P_{E v a l}$ could evaluate whether inlining increases register spills or bloats the code too much by comparing fragments in which calls were inlined and fragments in which they were not. This approach achieves a similar goal to inlining trials [11] but does not require the phases between $P_{i}$ and $P_{E v a l}$ to track any additional information. This application is interesting for embedded platforms where code size is critical.

- Procedure abstraction - in which the compiler factors out matching code sequences into a procedure [32] — is sometimes used to reduce code size. However code size might increase if the register allocator must spill around the call. Convergent cloning could choose the clone that did not have procedure abstraction applied if it ended up being smaller.

- The TRIPS compiler's back end estimates code size before splitting toolarge blocks, as Section 4.3 described. Sometimes its estimates are inaccurate, partly because the compiler performs some optimizations (e.g. peephole optimizations) after block splitting, and partly because the estimation is conservative in various ways. Convergent cloning could select an unsplit block if it ends up fitting within 128 instructions.

- Vectorizing compilers targeting SIMD instruction sets will often unroll a loop by 2 or 4 with the goal of converting groups of scalar operations into single SIMD instructions. Scalar code in the loop body can prevent this transformation from working, in which case no unrolling is probably preferable, but 
it is difficult to predict at loop unrolling time. Convergent cloning could discard any unrolled loops that failed to be vectorized.

The common theme to these uses is that $P_{i}$ transforms the code in a way that can result in better (faster and/or smaller) code, but puts stress on a limited machine resource such as registers or TRIPS block sizes. $P_{\text {Eval }_{i}}$ then evaluates whether the increased resource stress is just right or too much or if more stress could be tolerated. However, the increases in compile time require judicious selection and application of convergent optimizations.

\subsection{Just-In-Time and Interprocedural Compilation}

Convergent compilation can be used as-is in a just-in-time (JIT) compiler. However, many JIT compilers use staged dynamic optimization on frequently executed code, also called hotspot optimization. This mechanism offers an opportunity to improve the efficiency of self-evaluation by eliminating the unnecessary recompilation. Instead, the JIT compiler can piggyback the inner loop phases $P_{L B}$ and $P_{R B}$ onto its existing recompilation loop. This formulation would also complement Arnold et al.'s repository for combining online and offline profiling in a JIT by providing more accurate benefit measurements [13].

Convergent optimizations would operate on the JIT's existing recompilation unit (e.g., methods) along with its existing $P_{C P}$ and $P_{R B}$ phases. When $P_{\text {Eval }}$ evaluates $P_{i}$ 's decisions, rather than immediately rejecting substandard code, it records $F_{\text {info }}$ (e.g., code size, register spills, etc.) and executes this initial version of the code. If a fragment is hot and worth recompiling, $P_{R B}$ is invoked on it as usual, and $P_{i}$ can use $F_{\text {info }}$ to improve its heuristics during recompilation. This structure eliminates the additional compile-time cost that an ahead-oftime compiler must incur, requires no additional checkpointing cost (because it utilizes the JIT compiler's existing checkpointing mechanism), and still gains benefits for hot methods. The only addition required is the ability to record $F_{\text {info }}$ (which is typically compact) for each code fragment.

Another important consideration for convergent compilation is interprocedural analysis. If the fragment size is less than a whole module, any interprocedural analysis must take place during $\operatorname{pre}\left(P_{C P}\right)$ or $\operatorname{post}\left(P_{L B}\right)$; any analysis between $P_{C P}$ and $P_{L B}$ might be invalidated by the per-fragment re-running of phases. This requirement is unlikely to cause problems in practice.

\section{Conclusion}

We presented a general model of convergent compilation, in which a compiler adjusts its heuristic policies based on static self-evaluation. Implementing convergent compilation efficiently in an ahead-of-time compiler requires significant effort, but one can easily test if it will be worthwhile with the simple two-pass instance from Section 3.2. JIT compilers with multiple levels of optimization can easily piggybacking this approach the additional evaluation and heuristic tuning on to their existing recompilation frameworks. These characteristics are desirable in a field that is full of clever but complex ideas that do not make it into production compilers - as Arch Robison noted [33]: "Compile-time program 
optimizations are similar to poetry: more are written than actually published in production compilers."

This paper also identified previous instances of the static self-evaluation in the literature, showed how convergent compilation generalizes them, and described a number of additional optimization heuristics and phase orderings which could benefit from this approach. It illustrated effective convergent unrolling on the TRIPS architecture in which the compiler corrected its unrolling heuristic to meet the TRIPS block size constraints using a simple two pass instance. Furthermore, simulation results demonstrated that convergent unrolling reduces the number of executed blocks and improves performance.

\section{References}

1. Stephenson, M., Amarasinghe, S., Martin, M.C., O'Reilly, U.M.: Meta optimization: Improving compiler heuristics with machine learning. In: Proceedings of PLDI 2003, San Diego, CA (2003) 77-90

2. Stephenson, M., Amarasinghe, S.: Predicting unroll factors using supervised classification. In: The International Conference on Code Generation and Optimization, San Jose, CA (2005) 123-134

3. Cavazos, J., Moss, J.E.B.: Inducing heuristics to decide whether to schedule. In: Proceedings of PLDI 2004, Washington, DC (2004) 183-194

4. Agarwal, V., Hrishikesh, M., Keckler, S.W., Burger, D.: Clock rate versus IPC: The end of the road for conventional microarchitectures. In: Proceedings of the 27th International Symposium on Computer Architecture. (2000) 248-259

5. Kailas, K., Ebcioglu, K., Agrawala, A.K.: CARS: A new code generation framework for clustered ILP processors. In: International Symposium on High-Performance Computer Architecture. (2001) 133-143

6. Kessler, C., Bednarski, A.: Optimal integrated code generation for clustered VLIW architectures. In: Joint Conference on Languages, Compilers and Tools for Embedded Systems. (2002) 102-111

7. Zhong, H., Fan, K., Mahlke, S., Schlansker, M.: A distributed control path architecture for vliw processors. In: International Conference on Parallel Architectures and Compilation Techniques, Washingotn, DC (2005) 197-206

8. Burger, D., Keckler, S.W., McKinley, K.S., et al.: Scaling to the end of silicon with EDGE architectures. IEEE Computer (2004) 44-55

9. Smith, A., Burrill, J., Gibson, J., Maher, B., Nethercote, N., Yoder, B., Buger, D., McKinley, K.S.: Compiling for edge architectures. In: The International Conference on Code Generation and Optimization. (2006) 185-195

10. Callahan, D., Carr, S., Kennedy, K.: Improving register allocation for subscripted variables. In: Proceedings of PLDI 1990, White Plains, NY (1990) 53-65

11. Dean, J., Chambers, C.: Towards better inlining decisions using inlining trials. In: Proceedings of LFP '94, Orlando, FL (1994) 273-282

12. Brasier, T.S., Sweany, P.H., Carr, S., Beaty, S.J.: CRAIG: A practical framework for combining instruction scheduling and register assignment. In: International Conference on Parallel Architecture and Compiler Techniques, Cyprus (1995)

13. Arnold, M.R., Welc, A., Rajan, V.T.: Improving virtual machine performance using a cross-run profile repository. In: ACM Conference Proceedings on ObjectOriented Programming Systems, Languages, and Applications. (2005) 297-311

14. Moss, J.E.B., Utgoff, P.E., Cavazos, J., Precup, D., Stefanovic, D., Brodley, C., Scheeff, D.: Learning to schedule straight-line code. In: Neural Information Processing Systems - Natural and Synthetic, Denver, CO (1997) 
15. Yotov, K., Li, X., Ren, G., Cibulskis, M., DeJong, G., Garzaran, M.J., Padua, D., Pingali, K., Stodghill, P., Wu, P.: A comparison of empirical and model-driven optimization. In: Proceedings of PLDI 2003, San Diego, CA (2003) 63-76

16. Cavazos, J., Moss, J.E.B., O’Boyle, M.F.P.: Hybrid optimizations: Which optimization algorithm to use? In: International Conference on Compiler Construction, Vienna, Austria (2006)

17. Agakov, F., Bonilla, E., Cavazos, J., Franke, B., Fursin, G., O'Boyle, M.F.P., Thomson, J., Toussaint, M., Williams, C.K.I.: Using machine learning to focus iterative optimization. In: The International Conference on Code Generation and Optimization, New York, NY (2005) 295-305

18. Almagor, L., Cooper, K.D., Grosul, A., Harvey, T.J., Reeves, S.W., Subramanian, D., Torczon, L., Waterman, T.: Finding effective compilation sequences. In: Proceedings of LCTES 2004, Washington, DC (2004)

19. Cooper, K.D., Schielke, P.J., Subramanian, D.: Optimizing for reduced code space using genetic algorithms. In: Proceedings of LCTES '99, Atlanta (1999) 1-9

20. Haneda, M., Knijnenburg, P.M.W., Wijshoff, H.A.G.: Automatic selection of compiler options using non-parametric inferential statistics. In: International Conference on Parallel Architecture and Compiler Techniques, St. Louis, MO (2005) $123-132$

21. Ladd, S.R.: Acovea: Using natural selection to investigate software complexities (2003) http://www.coyotegulch.com/products/acovea/.

22. Triantafyllis, S., Vachharajani, M., August, D.I.: Compiler optimization-space exploration. In: The Journal of Instruction-level Parallelism. (2005) 1-25

23. Lau, J., Arnold, M., Hind, M., Calder, B.: Online performance auditing: Using hot optimizations without getting burned. In: ACM Conference on Programming Language Design and Implementation. (2006) 239-251

24. Alpern, B., Attanasio, D., Barton, J.J., et al.: The Jalapeño virtual machine. IBM System Journal 39 (2000)

25. Arnold, M., Fink, S., Grove, D., Hind, M., Sweeney, P.: A survey of adaptive optimization in virtual machines. IEEE Computer 93 (2005)

26. Smith, M.D.: Overcoming the challenges to feedback-directed optimization. In: Proceedings of Dynamo '00, Boston, MA (2000) 1-11

27. Sun MicroSystems: The Sun HotSpot compiler (2005) http://java-sun.com.products.hotspot.

28. Nagarajan, R., Sankaralingam, K., Burger, D., Keckler, S.W.: A design space evaluation of grid processor architectures. In: Proceedings of MICRO34, Austin, TX (2001) 40-53

29. Fisher, J.A.: Trace scheduling: A technique for global microcode compaction. IEEE Transactions on Computers C-30 (1981) 478-490

30. Mahlke, S.A., Lin, D.C., Chen, W.Y., Hank, R.E., Bringmann, R.A.: Effective compiler support for predicated execution using the hyperblock. In: Proceedings of MICRO25, Portland, OR (1992) 45-54

31. Maher, B., Smith, A., Burger, D., McKinley, K.S.: Merging head and tail duplication for convergent hyperblock formation. Technical Report TR-06-36, University of Texas at Austin (2006)

32. Baker, B.S.: Parameterized pattern matching: Algorithms and applications. Journal of Computer and System Sciences 52 (1996) 28-42

33. Robison, A.D.: Impact of economics on compiler optimization. In: Proceedings of the ACM 2001 Java Grande Conference, Palo Alto, CA (2001) 1-10 